'Oncología Médica, Unidad Funcional de Oncología Gastrointestinal, Instituto Nacional de Cancerología, México.

2Subdirección de Patología Instituto Nacional de Cancerología, México.

${ }^{3}$ Departamento de Cirugía Oncológica Gastrointestinal, Instituto Nacional de Cancerología, México.

Trabajo no recibió financiamiento. Los autores no declaran conflictos de interés.

Recibido el 2 de abril de 2018, aceptado el 23 de noviembre de 2018.

Correspondencia a:

Dr. Germán Calderillo Ruiz Unidad Funcional de Oncología Gastrointestinal. Instituto Nacional de Cancerología.

Teléfono: 5628-0400 ext 52101, 63200 . calderillo06@yahoo.com.mx

\section{Carcinoma epidermoide puro-combinado de vesícula biliar}

\author{
GERMÁN CALDERILLO-RUIZ1', ROBERTO HERRERA-GOEPFERT², \\ CONSUELO DÍAZ-ROMERO ${ }^{1}$, BERENICE CARBAJAL-LÓPEZ ${ }^{1,}$ \\ HORACIO LÓPEZ-BASAVE ${ }^{3}$
}

\section{Gallbladder epidermoid carcinoma}

Background: Gallbladder epidermoid carcinoma is rare and more common in women over 55 years of age. Aim: To report the features of 15 patients with gallbladder epidermoid carcinoma. Material and Methods: Review of medical records of patients with gallbladder cancer in an oncology service. Results: Of 207 patients with gallbladder cancer, 15 patients aged 53-72 years, 93\% women had an epidermoid component in their cancer. Forty percent were diabetic and 33\% had cholelithiasis. All had locoregional extension of the tumor. A cholecystectomy was done in nine patients (using open surgery in six). In six patients, only a biopsy was done. Median survival was 4.2 months. Conclusions: Gallbladder epidermoid carcinoma is uncommon and has a bad prognosis.

(Rev Med Chile 2018; 146: 1438-1443)

Key words: Carcinoma; Gallbladder; Prognosis; Survival.
11 1 carcinoma de la vesícula biliar es una neoplasia poco común en Europa y América del Norte, tiene una incidencia alta en algunas áreas de América Latina y Asia ${ }^{1,2}$. Los principales factores de riesgo incluyen: colelitiasis, obesidad, infecciones crónicas de la vesícula biliar, factores hormonales y la exposición ambiental a químicos específicos ${ }^{3}$. Se presenta más en mujeres mayores de 55 años ${ }^{4}$. Histológicamente, puede ser dividido en cuatro subtipos: 1) adenocarcinoma (con los subtipos papilar, tubular, mucinoso o en anillo de sello) (80 a 95\%); 2) carcinoma epidermoide (CE) y adenoescamoso (CAE) (2 a 10\%); 3) carcinoma indiferenciado (2 a 7\%) y otras neoplasias (carcinoma de células pequeñas, sarcomas, melanomas $y$ linfomas $)^{5-8}$.

Se presentan las características principales de 15 casos de carcinoma, incluyendo epidermoide (7) y adenoescamoso (8) atendidos en el servicio de Oncología Médica del Instituto Nacional de Cancerología, México.

\section{Material y Métodos}

Se realizó un estudio retrospectivo que incluyó una serie de 15 casos de pacientes con carcinoma epidermoide y adenoescamoso de la vesícula biliar. Se empleó el software SPSS para el cálculo de estadística descriptiva y el método de Kaplan-Meier para el cálculo de supervivencia global.

\section{Resultados}

De septiembre de 1998 a diciembre de 2016, se atendieron 207 pacientes con diagnóstico histológico de carcinoma (relación mujer:hombre de 4:1), de los cuales, 8 presentaban diagóstico de carcinoma epidermoide (CE) (Figuras 1 y 2 ) y 7 más con carcinoma adenoescamoso; los 15 casos de carcinomas con componente epidermoide correspondieron a $6,5 \%$ del total de los pacientes. 

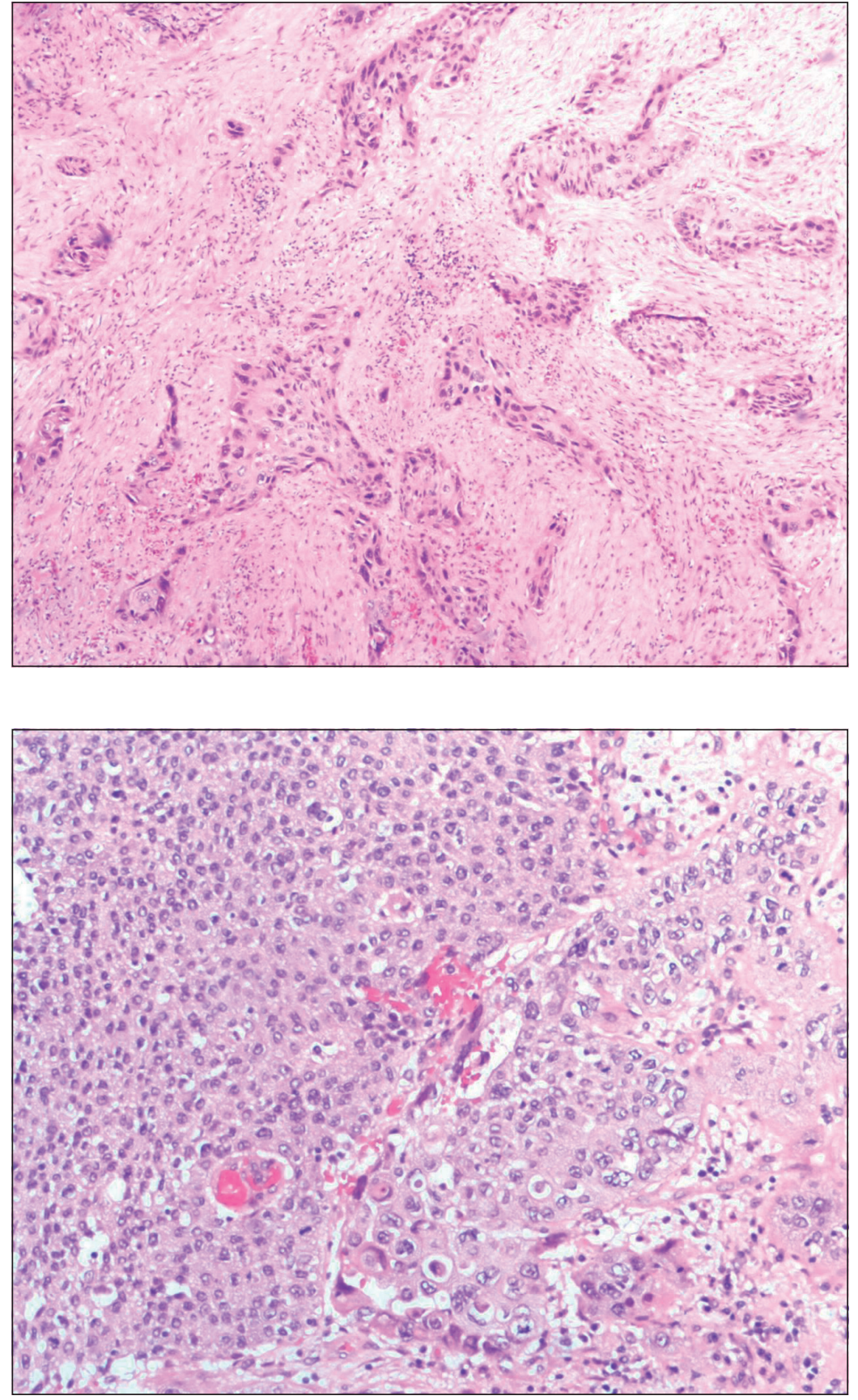

Figura 1. Se observan láminas de células neoplásicas que infiltran el espesor de la pared de la vesícula biliar (Hematoxilina y eosina, 4X).

Figura 2. Las células neoplásicas presentan núcleos pleomórficos con citoplasma eosinófilo abundante. Hay además, células con queratinización (Hematoxilina y eosina, 20X).

La Tabla 1 describe las características clínicas de los pacientes; $93,33 \%$ fueron mujeres con media de edad de 61,2 años (53-72). Dentro de los antecedentes, 6 pacientes presentaban diabetes mellitus, 7 hipertensión y 2 más diagnóstico previo de cáncer de mama. Cinco pacientes refirieron sa- ber que padecían de litiasis vesicular. Los síntomas más frecuentes fueron dolor en hipocondrio derecho $(73 \%)$, pérdida de peso (60\%), tumor palpable $(53 \%)$ y astenia $(40 \%)$. El tiempo transcurrido desde el inicio de los síntomas hasta el diagnóstico histológico fue de 5 meses (2-12 meses). 
Tabla 1. Características clínicas pacientes con carcinoma epidermoide de vesícula biliar

\begin{tabular}{|c|c|}
\hline Características & $\begin{array}{c}\text { Pacientes } \\
n=15(100 \%)\end{array}$ \\
\hline \multicolumn{2}{|l|}{ Sexo } \\
\hline Hombre & $1 \quad(6 \%)$ \\
\hline Mujer & $15(94 \%)$ \\
\hline \multicolumn{2}{|l|}{ Edad } \\
\hline Media (DS) & $61(14,5)$ \\
\hline \multicolumn{2}{|c|}{ Antecedentes personales patológicos } \\
\hline Diabetes mellitus tipo 2 & $6(40 \%)$ \\
\hline Hipertensión arterial & $7(46 \%)$ \\
\hline Otro tipo de cáncer (mama) & $2(14 \%)$ \\
\hline \multicolumn{2}{|l|}{${ }^{*} \mathrm{RT}$ mama derecha } \\
\hline \multicolumn{2}{|l|}{ Signos y síntomas } \\
\hline Dolor & $14(93 \%)$ \\
\hline Pérdida de peso & $9(60 \%)$ \\
\hline Astenia & $10(66 \%)$ \\
\hline Tumor palpable & $8(53 \%)$ \\
\hline Náuseas & $7(46 \%)$ \\
\hline
\end{tabular}

Los parámetros de laboratorio iniciales (Tabla 2 ), indican principalmente hipoalbuminemia (60\%), anemia (53\%) con niveles séricos menores de lo normal, mientras que en seis pacientes se observaron niveles anormales de antígeno carcinoembrionario (ACE) por arriba de $10 \mathrm{ng} / \mathrm{mL}$ y de Ca 19-9 (> 35 U/Ml).

En cuanto al procedimiento quirúrgico, se realizó colecistectomía en 9 pacientes, incluyendo laparoscópica (3) y cirugía abierta (6). El resto fue sometido a exploración quirúrgica con biopsia (6). Se efectuó resección multiorgánica (colcecistectomía y hemicolectomía derecha, hepatectomía parcial y resección de pared abdominal) en un paciente.

Se documentó enfermedad localmente avanzada en 2 casos y enfermedad metastásica en 13 casos con actividad pulmonar, en hígado y peritoneo por contigüidad, pulmón y peritoneo (Tabla 3 ).

A las 2 pacientes con enfermedad localmente avanzada se les ofreció cirugía y radioterapia alcanzando una supervivencia de 31 y 95 semanas. Ocho pacientes recibieron tratamiento paliativo con quimioterapia, incluyendo esquemas como capecitabina, gemcitabina y platino, Seis de ellos

Tabla 2. Datos de laboratorio al ingreso de las pacientes

\begin{tabular}{|c|c|c|c|c|c|}
\hline Caso & $\begin{array}{c}\text { Bilirrubina total } \\
\text { g/dL }\end{array}$ & $\begin{array}{l}\text { Albúmina } \\
\text { g/dL }\end{array}$ & $\begin{array}{c}\text { Hemoglobina } \\
\text { gr/dL }\end{array}$ & $\begin{array}{c}\text { ACE } \\
\mathbf{n g} / \mathbf{m L}\end{array}$ & $\begin{array}{c}\text { Ca19-9 } \\
\text { U/mL }\end{array}$ \\
\hline 1 & 6,83 & 3,5 & 14,2 & ND & 10,6 \\
\hline 2 & 5,8 & 3 & 9,8 & ND & ND \\
\hline 3 & ND & 2,3 & 11,3 & ND & 36,8 \\
\hline 4 & 0,4 & 2,7 & 10,3 & 3,5 & ND \\
\hline 5 & 0,3 & 2,5 & 9,8 & 26,1 & 42,2 \\
\hline 6 & 0,9 & 2,6 & 7,9 & 18,9 & 169 \\
\hline 7 & 0,7 & 3,4 & 10,9 & 30,6 & 2,5 \\
\hline 8 & 26,4 & 3,6 & 14 & 324 & 1914 \\
\hline 9 & 0,6 & 3,7 & 14,4 & 8,3 & 6 \\
\hline 10 & 0,7 & 3,1 & 13,9 & 93,8 & 13,8 \\
\hline 11 & 1,4 & 1,9 & 10,1 & 88,5 & 2932 \\
\hline 12 & ND & ND & 15,6 & ND & 4,02 \\
\hline 13 & 0,5 & 3,9 & 15,8 & 1,04 & 18,6 \\
\hline 14 & 0,4 & 2,4 & 9,3 & 4,47 & 30,3 \\
\hline 15 & 0,6 & 4 & 12,4 & 3,79 & 66,2 \\
\hline
\end{tabular}

ACE: Antígeno carcinoembrionario. ND: no determinado. GGT: Gama Glutamil Transferasa. DHL: Deshidrogenasa láctica. 
Tabla 3. Características quirúrgicas, otros tratamientos y resultado final

\begin{tabular}{|c|c|c|c|c|c|c|c|}
\hline & $\begin{array}{l}\text { Tipo } \\
\text { cirugía }\end{array}$ & Histología & Infiltración & QT & $\mathbf{R T}$ & Estado & $\begin{array}{c}\text { SV } \\
\text { (semanas) }\end{array}$ \\
\hline 1 & CCL-A & CE & LA & - & 35,5 Gy & Muerta & 31 \\
\hline 2 & CCL-A & CE & Ganglio, hígado & - & - & Muerta & 5 \\
\hline 3 & $\mathrm{LAPE}+\mathrm{B}$ & CE & Pulmón & Cape 1c & - & Muerta & 3 \\
\hline 4 & CCL-L & CE & Epiplón & Cape 2c & - & Muerta & 17 \\
\hline 5 & $\mathrm{LAPE}+\mathrm{B}$ & CE & Hígado & Gemci 1c & - & Muerta & 10 \\
\hline 6 & $L A P E+B$ & CE & Hígado & Gemci 1c & - & Muerta & 17 \\
\hline 7 & $\mathrm{LAPE}+\mathrm{B}$ & CE & Hígado, peritoneo & - & - & Muerta & 22 \\
\hline 8 & $\mathrm{LAPE}+\mathrm{B}$ & CE & Hígado, pulmones & - & & Muerta & 5 \\
\hline 9 & CCL-A & $\mathrm{AE}$ & LA & Gemci 1c & 35,5 Gy & Muerta & 95 \\
\hline 10 & CCL-A & $A E$ & Hígado & - & - & Muerta & 5 \\
\hline 11 & CCL-L & $\mathrm{AE}$ & $\begin{array}{c}\text { Ganglio cístico + litiasis } \\
\text { vesicular }\end{array}$ & - & - & Muerta & 10 \\
\hline 12 & CCL-A & $A E$ & Hígado, peritoneo & 5FULV 4c & 23 Gy 4F & Muerta & 38 \\
\hline 13 & CCL-L & $A E$ & Hígado & $\begin{array}{c}\text { GemOx 10c/ Gemci 7c/ } \\
\text { Paclitaxel } 2 c\end{array}$ & - & Muerta & 108 \\
\hline 14 & $L A P E+B$ & $A E$ & Hígado, peritoneo & Gemci 2c & - & Muerta & 24 \\
\hline 15 & CCL-A & $A E$ & Peritoneo, hígado & Gemox 8c/Cape 3c & - & Muerta & 36 \\
\hline
\end{tabular}

CCL: colecistectomía; B: biopsia; RMO: resección multiorgánica; CE: carcinoma epidermoide; AE: carcinoma adenoescamoso; QT: quimioterapia; RT: radioterapia; SV supervivencia; Cape: Capecitabina, Gemci: Gemcitabina; 5FULV: 5 Fluoracilo + Leucovorin; GemOx: Gemcitabina + Oxaliplatino.

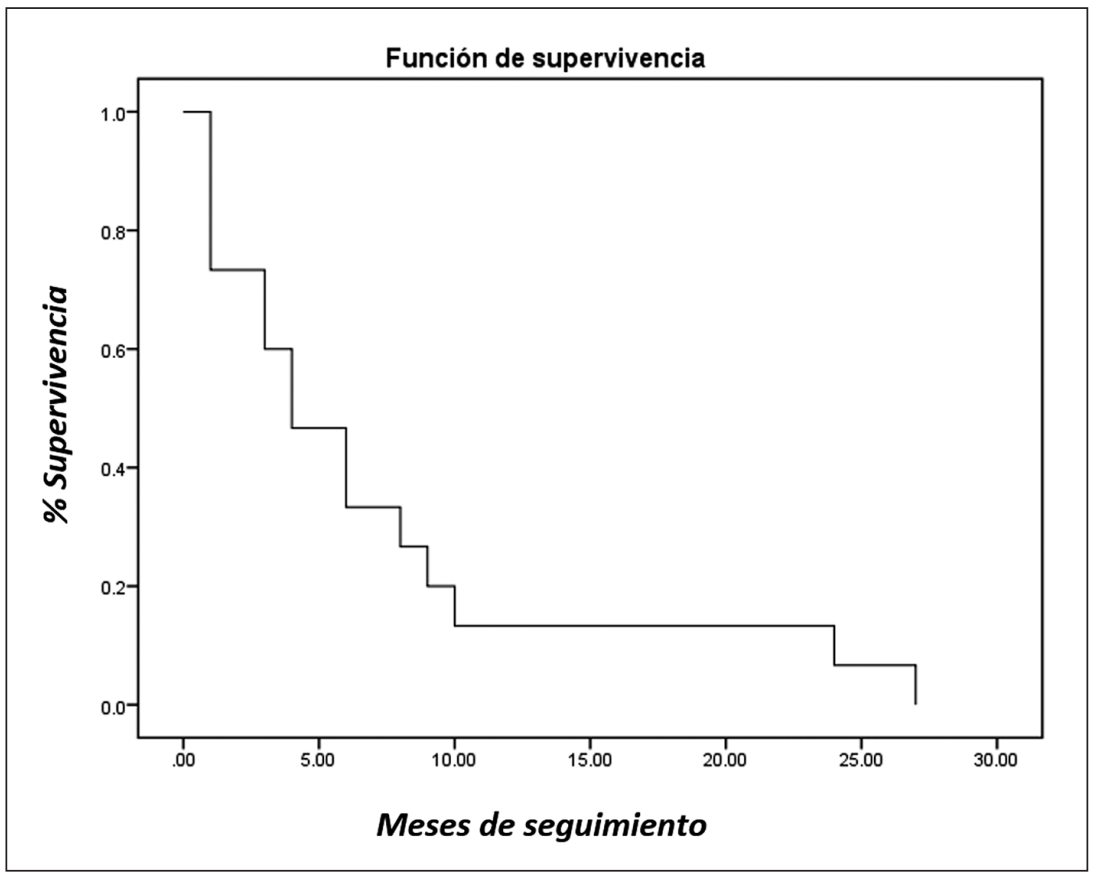

Figura 3. Curva de supervivencia pacientes carcinoma epidermoide. 
no presentaron beneficio debido a progresión tumoral con falla hepática. Las 2 pacientes restantes recibieron tratamiento paliativo por 8 y 10 ciclos, quienes alcanzaron enfermedad estable al cuarto y sexto ciclo del tratamiento respectivamente.

Se realizó el análisis de supervivencia global para todo el grupo en estudio, reportando una mediana de supervivencia global de 4 meses (HR 1,44 IC 95\% 1,16-6,84) (Figura 3).

\section{Discusión}

Las neoplasias malignas de la vesícula biliar ocupan el quinto lugar entre las neoplasias del tracto digestivo ${ }^{6}$. El carcinoma epidermoide y adenoescamoso se presentan más frecuentemente en mujeres con edad entre $40^{\mathrm{a}}$ y $70^{\mathrm{a}}$ década de la vida, con una proporción de 3:1 $1^{7-9}$; similar a la presentada en nuestro estudio 4:1 (M:H) con edad media de 60 años. Seis de los quince pacientes tenían diagnóstico de diabetes mellitus, enfermedad metabólica muy común en México que habitualmente está asociada con obesidad y sedentarismo.

Si bien no hay síntomas característicos para el carcinoma con diferenciación epidermoide, los más comunes en nuestra serie fueron dolor abdominal, pérdida de peso, tumor palpable y astenia, con un tiempo de evolución corto, lo que señala un crecimiento rápido. $\mathrm{Al}$ momento del diagnóstico generalmente se identifican niveles bajos de hemoglobina y albúmina en la mayoría de los pacientes ${ }^{7}$, como ocurrió en nueve y ocho de los casos presentados respecitvamente. El antígeno carcinoembrionario y el Ca 19-9 se observaron anormales en $6 / 15$ pacientes analizados. Estos marcados tumorales no son específicos de la neoplasia vesicular, y solo se relacionaron con la extensión de la enfermedad.

La litiasis vesicular es el principal factor de riesgo en estos carcinomas, así como el tiempo que se padece ${ }^{9,12}$; antecedente prevalente en 5/15 casos presentados en este estudio con 5 meses promedio de evolución.

El origen del carcinoma epidermoide en la vesícula biliar se desconoce, aunque la mayoría de los autores sugieren su origen a partir de una metaplasia pre-existente ${ }^{10}$. Otros consideran que se origina a partir de la diferenciación epidermoide de un adenocarcinoma previo, resultado de una expresión fenotípica mixta de las células neoplásicas ${ }^{7}$.

Las opciones terapéuticas dependerán de la extensión de la enfermedad, en etapas tempranas el tratamiento quirúrgico es recomendado, con colecistectomía y resección en bloque del tejido hepático adyacente en sospecha de infiltración. La hepato-pancreatoduodenectomía está justificada en caso de diseminación localizada sin metástasis o diseminación peritoneal; misma introducida como una opción, dado el tipo de diseminación observado ${ }^{13-15}$. En 10 pacientes se realizó colecistectomía y en 1 caso se practicó resección multiorgánica. El estudio BILCAP, demuestra el beneficio de la adyuvancia en la supervivencia global de pacientes con carcinoma de la vesícula biliar, sin embargo, incluye sólo 2 histologías: el adenocarcinoma y colangiocarcinoma sin incluir la histología de tipo epidermoide, por tal motivo, no se cuenta con un esquema de tratamiento adyuvante estándar para esta histología, sólo a manera de paliación ${ }^{16,18}$.

El EC Y AC presentan crecimiento agresivo con infiltración local en órganos adyacentes como hígado, estómago, duodeno y colon transverso, así como metástasis ganglionares regionales ocasionales $^{11}$. Se considera que el componente epidermoide posee una capacidad proliferativa mayor que el glandular ${ }^{10}$ y por tanto, su pronóstico es peor en comparación con el adenocarcinoma ${ }^{7}$. En este estudio los sitios más comunes de metástasis fueron hígado y epiplón (73\%).

La mayoría de los pacientes mueren alrededor de los 6 meses después del diagnóstico, en casos en los que no es posible realizar cirugía radical. Todos los pacientes presentados en esta serie fallecieron por actividad tumoral documentando una mediana de supervivencia de 4 meses, principalmente con disfunción hepatobiliar.

\section{Conclusiones}

El carcinoma de la vesícula biliar con componente epidermoide es raro y es más frecuente en mujeres mayores de 55 años. La resección quirúrgica es el tratamiento indicado en etapas iniciales. Dado que la mayoría de los pacientes se diagnostican con gran carga tumoral locorregional, el principal tratamiento es la paliación. El uso de quimioterapia y/o radioterapia debe ser considerado solo con fines paliativos. 


\section{Referencias}

1. Jemal A, Siegel R, Ward E, Hao Y, Xu J, Murray T, Thun MJ. Cancer statistics, 2008. CA Cancer J Clin 2008; 58 : 71-96.

2. Randi G, Franceschi S, La Vecchia C. Glallbladdrer cancer wordwide: geographical distribution and risk factors. Int J Cancer 2006; 118: 1591-602.

3. Lazcano-Ponce EC, Miguel JF, Muñoz N. Epidemiology and molecular pathology of gallbladder cancer. CA Cancer J Clin 2001; 51: 349-64.

4. Del Pozo AC, De Battista S, Velasco D, et al. Carcinoma epidermoide de vesícula: análisis de nuestra casuística. Acta Gastroenterol Latinoam 2005; 35: 162-4.

5. Henson DE, Albores-Saavedra J, Corle D: carcinoma of the gallbladder: histologic types, stage of disease, and survival rates. Cancer 1992; 70: 1493-7.

6. Sarbia M, Becker KF, Höfler H. Pathology of upper gastrointestinal malignancies. Semin Oncol 2004; 31: 465-75.

7. Kun-Ming Chan, Ming-Chin Yu, Wei-Chun Lee, et al. Adenosquamous/squamous cell carcinoma of the gallbladder. J Sur Oncol 2007; 95: 129-34.

8. Konstantinidis IT, Deshpande V, Genevay M, et al. Trends in presentation and survival for gallbladder cancer during a period of more than 4 decades. Arch Surg 2009; 144: 441-7.

9. Misra S, Chaturvedi A, Misra NC, et al. Carcinoma of the gallbladder. Lancet Oncol 2003; 4: 167-76.

10. Nishihara K, Nagai E, Izumi Y, et al. Adenosquamous carcinoma of the gallbladder: a clinicopatological, inmunohistochemical and flow cytometric study of twenty cases. Jpn J Cancer Res 1994; 85: 389-99.

11. Karasawa T, Itoh K, Komukai M, et al. Squamous cell carcinoma of the gallbladder. Report of two cases and review of literature. Acta Pathol Jpn 1981; 31: 299-308.

12. Cariati A, Cetta F. Squamous-cell and non-squamous-cell carcinoma of the gallbladder have different risk factors. Lancet Oncol 2003; 4: 393-4.

13. Muratore A, Polastri R, Bouzari H. Radical surgery for gallbladder cancer: a worthwhile operation? Eur J Surg Oncol 2000; 26: 160-3.

14. Khaira HS, Awad RW, Thompson AK. Squamous cell carcinoma of the gallbladder presenting with a biliary-colic fistula. Eur J Surg Oncol 1995; 21: 581-2.

15. Oohashi Y, Shirai Y, Wakai T, Nagakura S, Watanabe $\mathrm{H}$, Hatakeyama K. Adenosquamous carcinoma of the gallbladder warrants resection only if curative resection is feasible. Cancer 2002; 94 (11): 3000-5.

16. Miyazaki K, Tsutsumi N, Kitahara K, et al. Hepatopancreatoduodenectomy for squamous and adenosquamous carcinoma of the gallbladder. Hepato-Gastroenterol 1995; 42: 47-50.

17. Todoroki T, Kawamoto T, Otsuka M, et al. Benefits of combining radiotherapy with aggressive resection for stage IV gallbladder cancer. Hepatogastroenterology 1999; 46: 1585-91.

18. Primrose JN, Fox R, Palmer DH, Prasad R, Mirza D, Anthoney DA, et al. Adjuvant capecitabine for biliary tract cancer: The BILCAP randomized study 2017; 4006-6. 